\title{
Complex versus simple models: ion-channel cardiac toxicity prediction
}

\author{
Hitesh Mistry ${ }^{\text {Corresp. } 1}$ \\ 1 Division of Pharmacy, University of Manchester, Manchester, United Kingdom \\ Corresponding Author: Hitesh Mistry \\ Email address: hitesh.mistry@manchester.ac.uk
}

There is growing interest in applying detailed mathematical models of the heart for ionchannel related cardiac toxicity prediction. However, a debate as to whether such complex models are required exists. Here an assessment in the predictive performance between two established large-scale biophysical cardiac models and a simple linear model $B_{\text {net }}$ was conducted. Three ion-channel data-sets were extracted from literature. Each compound was designated a cardiac risk category using two different classification schemes based on information within CredibleMeds. The predictive performance of each model within each data-set for each classification scheme was assessed via a leave-one-out cross validation. Overall the $B_{\text {net }}$ model performed equally as well as the leading cardiac models in two of the data-sets and outperformed both cardiac models on the latest. These results highlight the importance of benchmarking complex versus simple models but also encourage the development of simple models. 
1

2

3

$4 \quad{ }^{1}$ Division of Pharmacy, University of Manchester, UK

5 Email correspondence: hitesh.mistry@manchester.ac.uk 


\section{Abstract}

7 There is growing interest in applying detailed mathematical models of the heart for ion-channel

8 related cardiac toxicity prediction. However, a debate as to whether such complex models are

9 required exists. Here an assessment in the predictive performance between two established large-

10 scale biophysical cardiac models and a simple linear model $B_{n e t}$ was conducted. Three ion-

11 channel data-sets were extracted from literature. Each compound was designated a cardiac risk

12 category using two different classification schemes based on information within CredibleMeds.

13 The predictive performance of each model within each data-set for each classification scheme

14 was assessed via a leave-one-out cross validation. Overall the $B_{n e t}$ model performed equally as

15 well as the leading cardiac models in two of the data-sets and outperformed both cardiac models

16 on the latest. These results highlight the importance of benchmarking complex versus simple

17 models but also encourage the development of simple models. 


\section{Introduction}

There is a growing belief within the pharmaceutical industry that in order to improve predictions of future experiments more detailed mathematical models of biology are required (Peterson \& Riggs, 2015; Knight-Schrijver et al., 2016). However by including more detail not only does the number of parameters that need to be estimated increase but so does the degree of structural uncertainty if the biology is not well understood i.e. the degree of confidence in the actual structure of the equations (Beven, 2005). The objective of this study is to look at this issue within the field of drug induced ion-channel cardiac toxicity. This area has a well-defined question relating to prediction where a debate about the complexity of the model needed is ongoing.

Numerous drugs were withdrawn from the market during the 1990s and early 2000s for causing a fatal arrhythmia, termed Torsades de Pointes (TdeP) (Yap \& Camm, 2003). Current pharmaceutical industry screening strategies on identifying these compounds at an early stage in drug development are based on the following biological insights (Antzelevitch \& Sicouri, 1994; Witchel, 2011). Prior to observing drug induced TdeP, prolongation of the QT interval is commonly seen within a patient. This prolongation is due to delayed repolarisation of cardiac cells within the ventricular wall, which is due to the drugs effect on the hERG ion-channel. Thus, the current approach in drug development involves screening a compounds effect against hERG in a high-throughput manner. However, there are other ion-channels involved in this process which the safety pharmacology community are now also screening compounds against (Colatsky et al., 2016). The question of interest then to the safety pharmacology community is: does measuring more than hERG improve prediction for TdeP, in humans?

In order to answer this question a clear definition of whether a compound has TdeP liabilities or not is required (Wiśniowska \& Polak, 2017). The first study to examine the association between multiple ion-channel inhibition and TdeP risk (Mirams et al., 2011) used a database created by AstraZeneca (Redfern et al., 2003). This database was built using literature data only and has never been updated since its initial publication. More recent studies (Kramer et al., 2013; Lancaster \& Sobie, 2016) have used the CredibleMeds database (Woosley, RL \& Romero, KA; Woosley et al., 2017) which was formerly known as AzCERT. Their classification is based on an extensive search of both the literature and public databases and is continuously updated in-light of new evidence. Furthermore it is recognised by the clinical community unlike the AstraZeneca database.

In terms of the modelling approach used the literature is divided in terms of the complexity required (Mistry, 2017). The complex models used are based on biophysical models which describe the changes in ionic currents over time within a single cardiac cell (Trayanova, 2011). They contain 100 s of parameters and 10s of differential equations. The drug input into these models involves scaling ion-channel conductance's by the amount of block at a given drug concentration (Brennan, Fink \& Rodriguez, 2009). Two biophysical models that have gained favour in the literature are the gold-standard, as described by Zhou et al. (Zhou et al., 2015), model by O'Hara et al. (O'Hara et al., 2011), herein referred to as ORD, which is being put forward for use by regulatory agencies (Colatsky et al., 2016) and another, by TenTusscher et al. 
58 (ten Tusscher \& Panfilov, 2006), forms a part of the cardiac safety simulator (Glinka \& Polak, 59 2015), herein referred to as TT. An alternative simpler mechanistic model being put forward 60 analyses the net difference, via a linear combination, in drug block of the ion-channels of interest, 61 termed $B_{n e t}$ (Mistry, 2017). In that study $B_{n e t}$ gave similar performance to a joint three biophysical 62 model/machine learning approach which used more than 300 metrics derived from the 63 biophysical models (Lancaster \& Sobie, 2016).

64 In this study the predictive performance of ORD, TT and $B_{n e t}$ models using a consistent and reliable definition of TdeP risk from CredibleMeds across three literature data-sets (Mirams et al., 2011; Kramer et al., 2013; Crumb Jr. et al., 2016) was analysed. Two of these data-sets, Mirams et al. (Mirams et al., 2011) and Kramer et al. (Kramer et al., 2013), measured drug effect against 3 ion-channels, hERG, Cav 1.2 and Nav 1.5 peak. The third and latest data-set, from Crumb et al. (Crumb Jr. et al., 2016), considers drug effect on 7 ion-channels, hERG (IKr), KCNQ1 + KCNE1 (IKs), Kv4.3 (Ito), Kir2.1 (IK1), Cav 1.2 (ICaL), Nav1.5 peak (INa) and Nav1.5 late (INaL), the largest number studied so far.

By using a consistent definition of TdeP risk across different data-sets that have different dimensionality in terms of ion-channels studied the analysis conducted will provide a detailed view on the performance of each model. Thus enabling scientists to make a more informed decision about which modelling approach is likely to be the most useful for the prediction problem considered.

\section{Methods}

78

79

\section{Data}

Ion-channel IC50 values, defined as concentration of drug that reduces the flow of current by 50\%, were collected from three publications (Mirams et al., 2011; Kramer et al., 2013; Crumb Jr. et al., 2016). Compounds within those data-sets were placed into two classification schemes based on the information in Credible Meds (Woosley, RL \& Romero, KA; Woosley et al., 2017), see Table 1. The first classification scheme termed QT/TdeP focusses on both QT prolongation and TdeP risk, which was used in two previous studies (Kramer et al., 2013; Lancaster \& Sobie, 2016). The second classification scheme focusses on known TdeP risk only. All data is provided in supplemental material.

\section{Model input data}

The percentage block against a given ion-channel inputted into all models was calculated using the mean maximal concentration observed corrected for plasma protein binding and is referred to as the effective therapeutic concentration (EFTPC), which was provided in the original articles, using a pore block model,

$$
\text { Block }=\frac{1}{1+\frac{I C 50}{E F T P C}}(1)
$$


92

93

94

95

96

97

98

99

100

101

102

103

104

105

106

107

108

109

110

111

112

113

114

115

116

117

118

119

\section{Models}

\section{Single cell cardiac model simulations}

The AP predict platform (Williams \& Mirams, 2015) which is a web-based cardiac modelling simulation platform (https://appredict.cs.ox.ac.uk) was used to simulate the ORD and TT models in all cases except for one simulation study. A MATLAB version of the ORD model available on the Rudylab website (http://rudylab.wustl.edu) was used when simulating the block of 7 ionchannels since that model on AP predict does not allow blocking of $\mathrm{INaL}$ - a current measured in the Crumb et al. data-set. The default settings within the AP predict platform were used i.e. $1 \mathrm{~Hz}$ pacing for 5 minutes with the APD90, time taken for the action potential to repolarise by $90 \%$, recorded using the last cycle. The same protocol was applied in MATLAB when exploring the 7 ion-channels within the ORD model i.e. $1 \mathrm{~Hz}$ pacing for 5 minutes with APD90 recorded using the last cycle. In all simulations drug block was initiated at the beginning of simulations.

\section{$\boldsymbol{B}_{\text {net }}$}

$B_{n e t}$ was defined as the net difference in block between repolarisation and depolarisation ionchannels as,

$$
\begin{gathered}
R_{i}-i \sum_{j=1}^{m} D_{j} \\
B_{\text {net }}=\sum_{i=1}^{n} i
\end{gathered}
$$

where $R_{i}$ and $D_{j}$ represent the percentage block against repolarisation and depolarisation ionchannels respectively for a specific drug. Ionic currents responsible for repolarisation are IKr IKs and Ito, and that for depolarisation are ICaL, INa (peak), INa (late) and IK1.

\section{Classification evaluation}

For each compound the percentage change in APD90 compared to control (no block) from the biophysical model simulations was recorded as was the $B_{n e t}$ value. These values were then placed within a logistic regression analysis to assess their correlative value to either QT/TdeP or TdeP risk. This was done via a leave one out cross validation (LOOCV). This involves training a classifier to $n-1$ compounds and testing on the $n^{\text {th }}$. Thus all compounds perform part of the testset. The predicted probability of risk for each test compound is then used to generate a ROC AUC (area under the receiver operating characteristic curve) which is reported. Note that LOOCV has been the method of choice within this field when assessing the correlation between metrics and drug risk (Mirams et al., 2011; Kramer et al., 2013; Lancaster \& Sobie, 2016).

\section{Results}




\section{Data}

122

The total number of compounds and their classification according to CredibleMeds across the 3 data-sets of interest can be seen in Figure 1. Although the total number of compounds differs from one data-set to another the proportions that are KR, PR and CR/NR does not appear to.

The distribution of block against each ionic current, at the EFTPC, across all data-sets can be seen in Figure 2. The plots show that the activity of the compounds is greatest against IKr across all data-sets. After IKr, ICaL appears to be the next channel for which a substantial amount of activity is seen. A somewhat surprising result is the degree of activity against INaL but not INa in the Crumb et al. data-set. The amount of activity against $\mathrm{INaL}$ in that data-set mirrors that of ICaL activity.

\section{Classification Evaluation}

The results of the leave-one-out cross validation for each data-set using various models for the two classification schemes can be seen in Tables 2 and 3. For the Mirams et al. data-set it's noticeable that ORD performs no better than using just block against hERG for either classification scheme. Furthermore for the QT/TdeP classification ORD is no better than random chance. Both TT and $B_{n e t}$ show a similar improvement over using just hERG block for both classification schemes.

Moving onto the Kramer et al. data-set the performance of all models improves dramatically over the Mirams et al. data-set. Here all 3 models show superior performance over just hERG block regardless of the classification scheme used. Note that again the performance of ORD is not as high as $B_{\text {net }}$ or TT. In addition the difference between $B_{n e t}$ and TT is negligible.

Within the latest data-set by Crumb et al. the performance of all models, when using only 3 ionchannels, drops to a level similar to that seen within the Mirams et al. data-set. The key difference between the results between those two data-sets is that ORD now shows similar performance to TT regardless of the classification scheme used. Furthermore neither biophysical model performs overly better than using hERG block. $B_{\text {net }}$ however appears to give reasonable performance again and appears to show an improvement over using hERG block for both classification schemes. Finally when moving onto using all the ion-channel data from the Crumb et al. data-set the difference in performance between the models is quite striking. $B_{n e t}$ 's performance improves with the addition of more information whereas there is little improvement in either biophysical model.

In summary the results show that the performance of the models is data-set dependent. However, within each data-set the $B_{\text {net }}$ model performs just as well if not better than leading biophysical models.

\section{Discussion}

There appears to be a strong belief within the field of ion-channel cardiac drug toxicity that large scale single cell (Mirams et al., 2011) and even whole heart models (Okada et al., 2015) are 
158 required to answer a well-defined question: does measuring more than hERG improve prediction 159 for TdeP, in humans? The evidence base, that suggests that large-scale biophysical models 160 perform better than simpler models for this question, simply does not exist. Previous studies have 161 shown that the performance of the large-scale cardiac models can be mirrored by simpler models 162 (Mistry, Davies \& Di Veroli, 2015; Mistry, 2017).

163 This study builds on those previous studies (Mistry, Davies \& Di Veroli, 2015; Mistry, 2017) of 164 comparing the performance of complex biophysical models versus simpler models in the 165 following way. First a consistent definition of TdeP risk based on the CredibleMeds database was 166 used across all data-sets (Woosley, RL \& Romero, KA; Woosley et al., 2017). Second in addition 167 to data-sets that considered drug activity against only 3 ion-channels (Mirams et al., 2011; 168 Kramer et al., 2013) a third data-set (Crumb Jr. et al., 2016) which measured drug affinity against 1697 ion-channels was also assessed.

170 The three models evaluated in this study were: 1) the gold-standard (Zhou et al., 2015) single cell model by O'Hara et al. (O'Hara et al., 2011); 2) the single cell model by TenTusscher et al. 172 (ten Tusscher \& Panfilov, 2006) which is used within the cardiac safety simulator (Glinka \& 173 Polak, 2015); 3) a linear mechanistic model evaluating the net difference in block between ion174 channels involved in repolarising and depolarising the action potential, $B_{\text {net }}$ (Mistry, 2017). Each 175 model was assessed via a leave-one-out cross validation using two different classification 176 schemes based on the CredibleMeds database. The first scheme focussed on the joint QT 177 prolongation and TdeP risk whereas the second scheme focussed on TdeP risk only. In addition to 178 using outputs from the aforementioned models within the classification exercise the amount of 179 block against hERG channel was used as a naïve benchmark.

180 Overall the analysis conducted showed that the performance of $B_{n e t}$ was superior to the more 181 complex cardiac models regardless of the classification scheme used. $B_{n e t}$ was also the only model that consistently showed the benefit of measuring more than hERG. Finally $B_{\text {net }}$ was the only model whose performance improved when moving from using information against 3 ion-channels to 7. These results may appear surprising but are not uncommon in prediction problems in other fields (Makridakis \& Hibon, 2000; Green \& Armstrong, 2015). The key reason why complex models are not necessarily more predictive than simpler models is due to model error i.e. error in the structure of the model itself (Beven, 2005). The concept of model error has only recently been assessed (Beattie et al., 2017) within the cardiac modelling field and so more needs to be done. Thus the effect of model error on predictivity is largely unknown, although in other fields it tends to dominate prediction uncertainty (Orrell et al., 2001; Refsgaard et al., 2006).

This study is not without its caveats. The first is that the data-sets used may be too small to understand how large a discrepancy there truly is between the different models. However it is hoped that by continuing to assess new data-sets as they become available that the community will eventually have a comprehensive compound list. Second, the latest data-set by Crumb et al.

195 (Crumb Jr. et al., 2016) although measured the affinity of drugs against 7 ion-channels the 196 compounds only really showed activity against 3. Thus, whether the results seen here will hold 197 for a set of compounds with activity against a large number of ion-channels still remains 
198 unknown. Similar to the previous caveat this can only be assessed as more data is generated. The 199 final caveat relates to the $B_{n e t}$ model itself. The model currently doesn't consider the kinetics of 200 blocking which has been highlighted as an important factor (Di Veroli et al., 2014). However 201 these studies have been on a small numbers of compounds and so a true assessment of the 202 importance of kinetics cannot be determined from those studies alone. If sufficient evidence 203 regarding the importance of drug kinetics does eventually become available adjustments to the $204 B_{\text {net }}$ model could be made.

\section{Conclusion}

206 In summary the study conducted here highlights the importance of benchmarking complex 207 models against simpler ones. Furthermore it highlights that simple mechanistic models can not 208 only give similar performance to large-scale mechanistic models but can out-perform them. 209 Finally it is hoped this study highlights that there is more than one solution to a problem and that 210 although the question and quality of data dictates the modelling approach it should not dictate the 211 size of the model.

\section{References}

Antzelevitch C., Sicouri S. 1994. Clinical relevance of cardiac arrhythmias generated by

afterdepolarizations. Role of $\mathrm{M}$ cells in the generation of $\mathrm{U}$ waves, triggered activity and torsade de pointes. Journal of the American College of Cardiology 23:259-277.

Beattie KA., Hill AP., Bardenet R., Cui Y., Vandenberg Jl., Gavaghan DJ., Boer TP de., Mirams GR. 2017.

Sinusoidal Voltage Protocols For Rapid Characterization Of Ion Channel Kinetics. bioRxiv:100677. DOI:

10.1101/100677.

Beven K. 2005. On the concept of model structural error. Water Science and Technology: A Journal of the International Association on Water Pollution Research 52:167-175.

Brennan T., Fink M., Rodriguez B. 2009. Multiscale modelling of drug-induced effects on cardiac electrophysiological activity. European Journal of Pharmaceutical Sciences: Official Journal of the

European Federation for Pharmaceutical Sciences 36:62-77. DOI: 10.1016/j.ejps.2008.09.013. 
227 Crumb Jr. WJ., Vicente J., Johannesen L., Strauss DG. 2016. An evaluation of 30 clinical drugs against the 228 comprehensive in vitro proarrhythmia assay (CiPA) proposed ion channel panel. Journal of

229 Pharmacological and Toxicological Methods 81:251-262. DOI: 10.1016/j.vascn.2016.03.009.

230 Di Veroli GY., Davies MR., Zhang H., Abi-Gerges N., Boyett MR. 2014. hERG Inhibitors with Similar Potency

231 But Different Binding Kinetics Do Not Pose the Same Proarrhythmic Risk: Implications for Drug Safety

232 Assessment. Journal of Cardiovascular Electrophysiology 25:197-207. DOI: 10.1111/jce.12289.

233 Glinka A., Polak S. 2015. QTc modification after risperidone administration - insight into the mechanism

234 of action with use of the modeling and simulation at the population level approach. Toxicology

235 Mechanisms and Methods 25:279-286. DOI: 10.3109/15376516.2015.1025346.

236 Green KC., Armstrong JS. 2015. Simple versus complex forecasting: The evidence. Journal of Business

237 Research 68:1678-1685. DOI: 10.1016/j.jbusres.2015.03.026.

238 Knight-Schrijver VR., Chelliah V., Cucurull-Sanchez L., Le Novère N. 2016. The promises of quantitative

239 systems pharmacology modelling for drug development. Computational and Structural Biotechnology

240 Journal 14:363-370. DOI: 10.1016/j.csbj.2016.09.002.

241 Kramer J., Obejero-Paz CA., Myatt G., Kuryshev YA., Bruening-Wright A., Verducci JS., Brown AM. 2013.

242 MICE models: superior to the HERG model in predicting Torsade de Pointes. Scientific Reports 3:2100.

243 DOI: 10.1038/srep02100.

244 Lancaster MC., Sobie E. 2016. Improved Prediction of Drug-Induced Torsades de Pointes Through

245 Simulations of Dynamics and Machine Learning Algorithms. Clinical Pharmacology \& Therapeutics:n/a-

246 n/a. DOI: 10.1002/cpt.367.

247 Makridakis S., Hibon M. 2000. The M3-Competition: results, conclusions and implications. International

248 Journal of Forecasting 16:451-476. DOI: 10.1016/S0169-2070(00)00057-1.

249 Mirams GR., Cui Y., Sher A., Fink M., Cooper J., Heath BM., McMahon NC., Gavaghan DJ., Noble D. 2011.

250 Simulation of multiple ion channel block provides improved early prediction of compounds' clinical

251 torsadogenic risk. Cardiovascular Research 91:53-61. DOI: 10.1093/cvr/cvr044. 
252 Mistry H. 2017. Complexity vs. Simplicity: The Winner Is? Clinical Pharmacology \& Therapeutics 101:326253 326. DOI: 10.1002/cpt.503.

254 Mistry HB., Davies MR., Di Veroli GY. 2015. A new classifier-based strategy for in-silico ion-channel cardiac 255 drug safety assessment. Frontiers in Pharmacology 6:59. DOI: 10.3389/fphar.2015.00059.

256 O'Hara T., Virág L., Varró A., Rudy Y. 2011. Simulation of the Undiseased Human Cardiac Ventricular 257 Action Potential: Model Formulation and Experimental Validation. PLoS Comput Biol 7:e1002061. DOI: 258 10.1371/journal.pcbi.1002061.

259 Okada J., Yoshinaga T., Kurokawa J., Washio T., Furukawa T., Sawada K., Sugiura S., Hisada T. 2015.

260 Screening system for drug-induced arrhythmogenic risk combining a patch clamp and heart simulator.

261 Science Advances 1:e1400142. DOI: 10.1126/sciadv.1400142.

262 Orrell D., Smith L., Barkmeijer J., Palmer TN. 2001. Model error in weather forecasting. Nonlin. Processes 263 Geophys. 8:357-371. DOI: 10.5194/npg-8-357-2001.

264 Peterson M., Riggs M. 2015. FDA Advisory Meeting Clinical Pharmacology Review Utilizes a Quantitative 265 Systems Pharmacology (QSP) Model: A Watershed Moment? CPT: Pharmacometrics \& Systems 266 Pharmacology 4:189-192. DOI: 10.1002/psp4.20.

267 Redfern WS., Carlsson L., Davis AS., Lynch WG., MacKenzie I., Palethorpe S., Siegl PKS., Strang I., Sullivan 268 AT., Wallis R., Camm AJ., Hammond TG. 2003. Relationships between preclinical cardiac 269 electrophysiology, clinical QT interval prolongation and torsade de pointes for a broad range of drugs: 270 evidence for a provisional safety margin in drug development. Cardiovascular Research 58:32-45. 271 Refsgaard JC., van der Sluijs JP., Brown J., van der Keur P. 2006. A framework for dealing with uncertainty 272 due to model structure error. Advances in Water Resources 29:1586-1597. DOI:

273 10.1016/j.advwatres.2005.11.013.

274 Trayanova NA. 2011. Whole-Heart Modeling Applications to Cardiac Electrophysiology and 275 Electromechanics. Circulation Research 108:113-128. DOI: 10.1161/CIRCRESAHA.110.223610. 
276 ten Tusscher KHWJ., Panfilov AV. 2006. Alternans and spiral breakup in a human ventricular tissue model.

277 American Journal of Physiology. Heart and Circulatory Physiology 291:H1088-1100. DOI:

278 10.1152/ajpheart.00109.2006.

279 Williams G., Mirams GR. 2015. A web portal for in-silico action potential predictions. Journal of

280 Pharmacological and Toxicological Methods 75:10-16. DOI: 10.1016/j.vascn.2015.05.002.

281 Wiśniowska B., Polak S. 2017. Am I or am I not proarrhythmic? Comparison of various classifications of 282 drug TdP propensity. Drug Discovery Today 22:10-16. DOI: 10.1016/j.drudis.2016.09.027.

283 Witchel HJ. 2011. Drug-induced hERG Block and Long QT Syndrome. Cardiovascular Therapeutics 29:251-

284 259. DOI: 10.1111/j.1755-5922.2010.00154.x.

285 Woosley, RL, Romero, KA www.Crediblemeds.org.

286 Woosley RL., Romero K., Heise CW., Gallo T., Tate J., Woosley RD., Ward S. 2017. Adverse Drug Event

287 Causality Analysis (ADECA): A Process for Evaluating Evidence and Assigning Drugs to Risk Categories for

288 Sudden Death. Drug Safety 40:465-474. DOI: 10.1007/s40264-017-0519-0.

289 Yap YG., Camm AJ. 2003. Drug induced QT prolongation and torsades de pointes. Heart 89:1363-1372.

290 Zhou X., Bueno-Orovio A., Orini M., Hanson B., Hayward MP., Taggart P., Lambiase PD., Burrage K.,

291 Rodriguez B. 2015. In Vivo and In Silico Investigation into Mechanisms of Frequency Dependence of

292 Repolarization Alternans in Human Ventricular Cardiomyocytes. Circulation

293 Research:CIRCRESAHA.115.307836. DOI: 10.1161/CIRCRESAHA.115.307836. 


\section{Figure Legends}

295 Figure 1: Stacked bar-chart shows the proportion of compounds in each data-set that are KR, PR 296 or CR/NR based on information within the CredibleMeds database.

297 Figure 2: Boxplots show the distribution of block for each ionic current across all 3 data-sets.

298 Table Titles

299 Table 1: Description of the two classification schemes constructed from the CredibleMeds 300 database.

301 Table 2: ROC AUC values from the leave one out cross validation for assessing the joint 302 QT/TdeP risk across all data-sets for all models considered.

303 Table 3: ROC AUC values from the leave one out cross validation ROC AUC for assessing TdeP 304 risk only across all data-sets for all models considered. 


\section{Figure 1 (on next page)}

Stacked bar-chart shows the proportion of compounds in each data-set that are KR, PR or CR/NR based on information within the CredibleMeds database. 
Figure 2 (on next page)

Boxplots show the distribution of block for each ionic current across all 3 data-sets. 


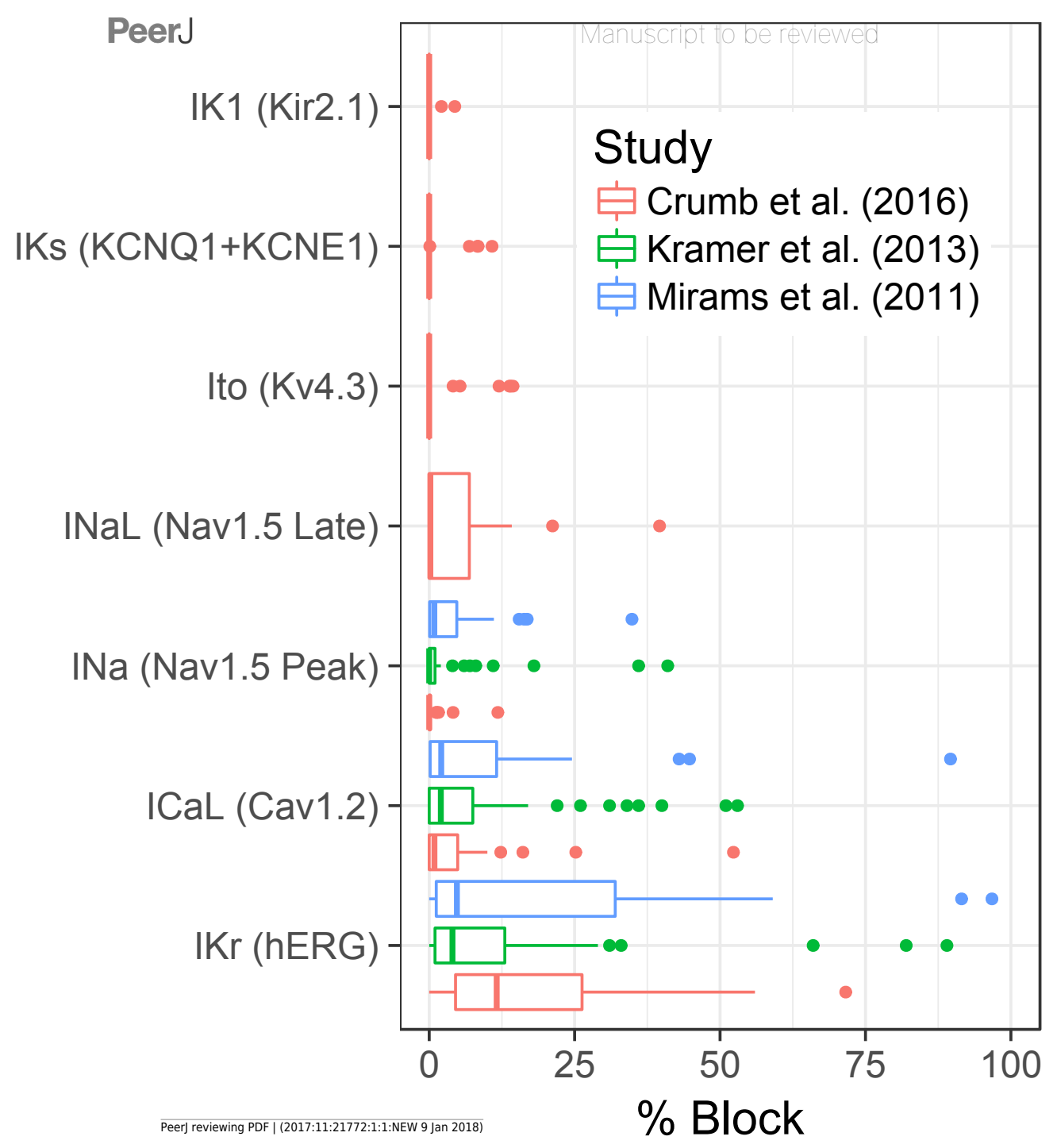




\section{Table $\mathbf{1}$ (on next page)}

Description of the two classification schemes constructed from the CredibleMeds database. 


\begin{tabular}{llcc}
\multicolumn{1}{c}{ CredibleMeds } & \multicolumn{1}{c}{ Description } & QT/TdeP & TdeP \\
Known Risk (KR) & Known TdeP Risk & +ive & +ive \\
Possible Risk (PR) & Known QT Risk & +ive & -ive \\
Conditional Risk (CR) & Conditional TdeP Risk (e.g. drug-drug interaction) & -ive & -ive \\
No Risk (NR) & Not listed on CredibleMeds & -ive & -ive \\
\hline
\end{tabular}

1 


\section{Table 2 (on next page)}

ROC AUC values from the leave one out cross validation for assessing the joint QT/TdeP risk across all data-sets for all models considered. 


\begin{tabular}{|c|c|c|c|c|}
\hline \multicolumn{5}{|c|}{ Leave One Out Cross Validation ROC AUC } \\
\hline \multirow[t]{2}{*}{ Data-Set } & \multicolumn{3}{|c|}{3 ion-channels } & hERG \\
\hline & $B_{\text {net }}$ & ORD: $\triangle$ APD90 & TT: $\triangle \mathrm{APD} 90$ & $\%$ Block IKr \\
\hline $\begin{array}{c}\text { Mirams } \\
\text { (2011) }\end{array}$ & 0.71 & 0.53 & 0.68 & 0.51 \\
\hline $\begin{array}{c}\text { Kramer } \\
(2013)\end{array}$ & 0.96 & 0.86 & 0.94 & 0.67 \\
\hline $\begin{array}{l}\text { Crumb } \\
(2016)\end{array}$ & 0.71 & 0.65 & 0.65 & 0.61 \\
\hline & & 7 ion-chann & & \\
\hline $\begin{array}{l}\text { Crumb } \\
\text { (2016) }\end{array}$ & 0.82 & 0.67 & $0.60 *$ & \\
\hline
\end{tabular}

1 *based on 6 ion-channels - INaL not modelled by TenTusscher et al. (TT); $\triangle A P D 90$ : percentage change in 2 APD90 


\section{Table 3(on next page)}

ROC AUC values from the leave one out cross validation ROC AUC for assessing TdeP risk only across all data-sets for all models considered. 


\begin{tabular}{|c|c|c|c|c|}
\hline \multicolumn{5}{|c|}{ Leave One Out Cross Validation ROC AUC } \\
\hline \multirow[t]{2}{*}{ Data-Set } & \multicolumn{3}{|c|}{3 ion-channels } & hERG \\
\hline & $B_{\text {net }}$ & ORD: $\triangle$ APD90 & TT: $\triangle \mathrm{APD} 90$ & $\%$ Block IKr \\
\hline $\begin{array}{c}\text { Mirams } \\
\text { (2011) }\end{array}$ & 0.78 & 0.66 & 0.75 & 0.62 \\
\hline $\begin{array}{c}\text { Kramer } \\
(2013)\end{array}$ & 0.86 & 0.80 & 0.84 & 0.68 \\
\hline $\begin{array}{l}\text { Crumb } \\
(2016)\end{array}$ & 0.68 & 0.61 & 0.62 & 0.57 \\
\hline & & 7 ion-chann & & \\
\hline $\begin{array}{l}\text { Crumb } \\
\text { (2016) }\end{array}$ & 0.77 & 0.63 & $0.59 *$ & \\
\hline
\end{tabular}

1 *based on 6 ion-channels - INaL not modelled by TenTusscher et al. (TT); $\triangle A P D 90$ : percentage change in 2 APD90 